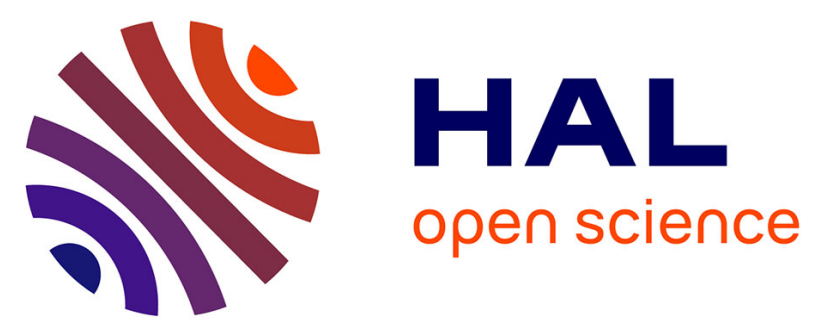

\title{
Impact of a centre and home-based cardiac rehabilitation program on the quality of life of teenagers and young adults with congenital heart disease: The QUALI-REHAB study rationale, design and methods
}

Pascal Amedro, Arthur Gavotto, Antoine Legendre, Kathleen Lavastre, Charlène Bredy, Gregoire de La Villeon, Stefan Matecki, d'Arcy Vandenberghe, Manon Ladeveze, Fanny Bajolle, et al.

\section{- To cite this version:}

Pascal Amedro, Arthur Gavotto, Antoine Legendre, Kathleen Lavastre, Charlène Bredy, et al.. Impact of a centre and home-based cardiac rehabilitation program on the quality of life of teenagers and young adults with congenital heart disease: The QUALI-REHAB study rationale, design and methods. International Journal of Cardiology, 2018, 10.1016/j.ijcard.2018.12.050 . hal-01965493

\author{
HAL Id: hal-01965493 \\ https://hal.science/hal-01965493
}

Submitted on 14 May 2020

HAL is a multi-disciplinary open access archive for the deposit and dissemination of scientific research documents, whether they are published or not. The documents may come from teaching and research institutions in France or abroad, or from public or private research centers.
L'archive ouverte pluridisciplinaire HAL, est destinée au dépôt et à la diffusion de documents scientifiques de niveau recherche, publiés ou non, émanant des établissements d'enseignement et de recherche français ou étrangers, des laboratoires publics ou privés. 


\section{Impact of a centre and home-based cardiac rehabilitation program on the quality of life of teenagers and young adults with congenital heart disease: The QUALI-REHAB study rationale, design and methods}

Pascal Amedro a,b,*, Arthur Gavotto ${ }^{\mathrm{a}, \mathrm{c}}$, Antoine Legendre ${ }^{\mathrm{d}}$, Kathleen Lavastre ${ }^{\mathrm{a}}$, Charlene Bredy ${ }^{\mathrm{a}, \mathrm{s}}$, Gregoire De La Villeon ${ }^{\mathrm{a}, \mathrm{c}}$, Stefan Matecki ${ }^{\mathrm{a}, \mathrm{b}}$, D'Arcy Vandenberghe ${ }^{\mathrm{a}}$, Manon Ladeveze ${ }^{\mathrm{a}}$, Fanny Bajolle ${ }^{\mathrm{d}}$, Gilles Bosser ${ }^{\mathrm{e}}$, Helene Bouvaist ${ }^{\mathrm{f}}$, Philippe Brosset ${ }^{\mathrm{g}}$, Laurence Cohen ${ }^{\mathrm{h}}$, Sarah Cohen ${ }^{\mathrm{i}}$, Sonia Corone ${ }^{\mathrm{j}}$, Claire Dauphin ${ }^{\mathrm{k}}$, Yves Dulac ${ }^{1}$, Sebastien Hascoet ${ }^{\mathrm{i}}$, Xavier Iriart $^{\mathrm{m}}$, Magalie Ladouceur ${ }^{\mathrm{n}}$, Loic Mace ${ }^{\mathrm{o}}$, Oxana-Anca Neagu ${ }^{\mathrm{p}}$, Caroline Ovaert ${ }^{\mathrm{o}, \mathrm{u}}$, Marie-Christine Picot ${ }^{\mathrm{q}}$, Laurent Poirette ${ }^{\mathrm{r}}$, Frederique Sidney ${ }^{\mathrm{s}}$, Camille Soullier ${ }^{\mathrm{t}}$, Jean-Benoit Thambo ${ }^{\mathrm{m}}$, Nicolas Combes ${ }^{\mathrm{v}}$, Damien Bonnet ${ }^{\mathrm{d}}$, Sophie Guillaumont ${ }^{\mathrm{a}, \mathrm{c}}$

a Paediatric and Congenital Cardiology Department, M3C Regional Reference CHD Centre, CHU Montpellier, Montpellier, France

${ }^{\mathrm{b}}$ PhyMedExp, CNRS, INSERM, University of Montpellier, Montpellier, France

' Paediatric Cardiology and Rehabilitation Centre, Institut-Saint-Pierre, Palavas, Les-Flots, France

' Paediatric Cardiology Department, AP-HP, Necker-Enfants malades, M3C National Reference CHD Centre, Paris Descartes University, Sorbonne Paris Cite, Paris, France

e Paediatric Cardiology Department, Children's Hospital, M3C Regional Reference CHD Centre, University Hospital of Nancy, Vandoeuvre-les-Nancy, France

${ }^{\mathrm{f}}$ Department of Cardiology, M3C Regional Reference CHD Centre, CHU Grenoble, France

${ }^{\mathrm{g}}$ Mother and Children Hospital, $\mathrm{CHU}$ Limoges, France

${ }^{\text {h }}$ CHD private medical office, Massy, France

i Paris-Sud Faculty of Medicine, INSERM U999, Marie-Lannelongue Hospital, M3C National Reference CHD Centre, Paris-Sud University, Paris, Saclay, Le Plessis-Robinson, France

${ }^{\mathrm{j}}$ Cardiac Rehabilitation Centre, Hospital of Bligny, Bris-sous-Forge, France

k Department of Cardiology, M3C Regional Reference CHD Centre, Gabriel Montpied University Hospital, Clermont-Ferrand, France

${ }^{1}$ Paediatric Cardiology Unit, Children's Hospital, M3C Regional Reference CHD Centre, CHU Toulouse, France

m Paediatric and Adult Congenital Heart Disease Department, Hôpital Cardiologique du Haut-Lévêque, M3C National Reference CHD Centre, CHU de Bordeaux, Bordeaux, Pessac, France

${ }^{\mathrm{n}}$ Adult Congenital Heart Disease Unit, Department of Cardiology, European Hospital Georges Pompidou, M3C National Reference CHD Centre, Paris, France

o Paediatric and Congenital Medico-Surgical Cardiology Department, M3C Regional Reference CHD Centre, AP-HM, La Timone University Hospital, Marseille, France

p Cardiac Rehabilitation Centre, Beaumont-de-Lomagne, France

${ }^{\mathrm{a}}$ Department of Epidemiology and Biostatistics, CHU Montpellier, France

${ }^{\mathrm{r}}$ Leon Berard Hospital, Cardiac Rehabilitation Centre, Hyeres, France

${ }^{s}$ Cardiac Rehabilitation Centre, Fontfroide Clinic, Montpellier, France

${ }^{\mathrm{t}}$ Cardiology Department, Caremeau University Hospital, Nimes, France

" Laboratory of Medical Genetics, INSERM UMR 1251, Aix-Marseille University, Marseille, France

v Department of Cardiology, Clinique Pasteur, Toulouse, France

Keywords:

Cardiac rehabilitation

Congenital heart defect

Health-related quality of life

Cardiovascular deconditioning

Health education

Secondary prevention

\section{A B S T R A C T}

Background: Advances in congenital heart disease (CHD) have transferred the mortality from childhood to adulthood. Exercise capacity in young patients with $\mathrm{CHD}$ remains lower than in the general population, resulting in deconditioning and impaired quality of life. Evidence based-medicine in cardiac rehabilitation in this age group with CHD remains limited. We present the QUALI-REHAB study rationale, design and methods.

Methods: The QUALI-REHAB trial is a nationwide, multicentre, randomised, controlled study, aiming to assess the impact of a combined centre and home-based cardiac rehabilitation program on the quality of life of adolescents and young adults ( 13 to 25 years old) with CHD. Patients with a maximum oxygen uptake ( $\left.\mathrm{VO} 2_{\max }\right)<80 \%$ and/or a ventilatory anaerobic threshold (VAT) $<55 \%$ of predicted $\mathrm{VO} 2_{\max }$, will be eligible. Patients will be randomised into 2 groups (12-week cardiac rehabilitation program vs. controls). The primary outcome is the change in the PedsQL quality of life score between baseline and 12-month follow-up. A total of 130 patients are required to observe a significant increase of $7 \pm 13.5$ points in the PedsQL, with a power of $80 \%$ and an alpha risk of $5 \%$. The secondary outcomes are: $\mathrm{VO}_{\mathrm{max}}$, VAT, stroke volume, clinical outcomes, physical and psychological status, safety and acceptability.

is The authors take responsibility for all aspects of the reliability and freedom from bias of the data presented and their discussed interpretation.

* Corresponding author at: Paediatric and Congenital Cardiology Department, Montpellier University Hospital, 371 Avenue du Doyen Giraud, 34295 Montpellier, France. E-mail address: p-amedro@chu-montpellier.fr (P. Amedro). 
Conclusion: After focusing on the survival in CHD, current research is opening on secondary prevention and patient-related outcomes. The QUALI-REHAB trial intends to assess if a combined centre and home-based rehabilitation program, could improve the quality of life and the exercise capacity in youth with CHD.

Trial registration: Clinicaltrials.gov (NCT03690518).

\section{Introduction}

In the past three decades, tremendous progress in paediatric cardiology has significantly improved the overall prognosis, and, consequently, transferred the mortality in congenital heart disease (CHD) from childhood to adulthood [1]. As a result, after focusing on the survival of children with CHD, more attention is being given to health-related quality of life and secondary prevention [2].

Indeed, from a large European multicentre research program, we found that the quality of life of children and young adults with CHD remained significantly lower than that of the general paediatric population, especially when considering their physical well being [3]. Moreover, from a contemporary paediatric cohort of nearly 800 subjects, we recently showed that the exercise capacity, as assessed by the maximum oxygen uptake $\left(\mathrm{VO}_{\max }\right)$, was moderately but significantly lower in children with CHD than in healthy matched children, and we observed a mean overall $\mathrm{VO} 2_{\max }$ decline of $2 \%$ per year in this population [4]. We also demonstrated, for the first time in a paediatric population, that the $\mathrm{VO}_{\text {max }}$ of children with CHD correlated with their quality of life [5].

The contemporary generation of children with CHD is supposedly not limited in submaximal physical activity, when considering their ventilatory anaerobic threshold (VAT) [6]. However, the VAT is much less correlated to their quality of life than is the $\mathrm{VO}_{\max }[5]$ and we showed that physical deconditioning associated with impaired VAT affected three times more children with CHD than controls, even for simple CHD [4]. As a result, many children with CHD suffer from an unpleasant feeling of exercise-induced dyspnoea, and, cumulated with social barriers to physical activity, often "remain on the side-line" at school or in their social life [7]. Despite the promotion of physical activity in the current guidelines $[8,9]$, many adolescents and young adults with CHD are entrapped in the vicious circle of deconditioning, and, ultimately, exposed to the consequences of a sedentary lifestyle: overweight, high blood pressure, social exclusion, and impaired quality of life [10-12]. Conversely, CHD patients who have been physically active since childhood are less likely to become sedentary adults [13].

In acquired cardiac disease, physical inactivity stands as a significant cardiovascular risk factor, and cardiac rehabilitation has been proven to reduce cardiac morbidity and improve quality of life [14]. Therefore, cardiac rehabilitation currently belongs to the standard of care in adult patients with chronic heart failure [15]. Yet, European research on implementation of rehabilitation programs indicated that only a minority of eligible patients with heart failure were receiving appropriate exercise training, for various reasons (lack of medical and paramedical resources, patients' difficulties with regular attendance at the local hospital, and reluctance to join group-based classes) [16]. Therefore, modern home-based rehabilitation programs have been designed to overcome these suboptimal participation rates [17].

Despite a lower level of evidence than in adult acquired heart failure, the beneficial effects of cardiac rehabilitation in patients with CHD have also been highlighted [18-21]. The meta-analysis from Duppen et al. included 621 patients aged 4 to 45 years from 31 studies, and underlined the positive effects of cardiac rehabilitation programs in the CHD population [22]. Most studies used a 12-week rehabilitation program, with an average of 3 exercise sessions per week, and mostly relied on centre-based training or non-supervised home-based training [22]. These beneficial effects were found on simple [18] and complex CHD, such as tetralogy of Fallot $[20,23]$, single ventricle [23], and systemic right ventricle [24]. Similarly, Corina et al. reported that rehabilitation increased muscle mass, ventricular ejection fraction and cardiac output in patients with a Fontan circulation [25]. Moreover, the randomised study from Winter et al. found a significant $7 \%$ increase in $\mathrm{VO}_{\max }$ after 10 weeks of exercise training in patients with a systemic right ventricle, [26]. Overall, rehabilitation programs in patients with CHD appear to be useful and safe, even in children [22,27]. Indeed, the meta-analysis from Gomes-Neto et al. studied 8 paediatric studies (age range from 11 to 16 years) with various sample sizes ( $n=14$ to 83 ), suggesting a beneficial effect of cardiac rehabilitation in children with CHD, in terms of $\mathrm{VO}_{\max }$ (mean increase of 13\%), quality of life, psychological well-being, and muscular strength [21].

Nevertheless, clinical research on cardiac rehabilitation remains limited in the young CHD population [21,22,27]. Currently, consistent data are needed from multicentre randomised studies relying on relevant outcomes, such as quality of life and $\mathrm{VO}_{\max }$, and using modern rehabilitation programs adapted to the generation of youth with CHD.

In the QUALI-REHAB trial, we aim to assess the impact of a combined centre and home-based cardiac rehabilitation program on the quality of life of adolescents and young adults with CHD, through a nationwide multicentre randomised trial. We also intend to evaluate, in this population, the impact of the rehabilitation program on clinical, psychosocial and exercise capacity outcomes.

\section{Methods}

\subsection{Study design}

The QUALI-REHAB trial is a prospective, multicentre, randomised, controlled, parallel arm study, with a follow-up of 12 months and expected recruitment duration of 36 months.

Randomisation will be centralised, using a 1:1 ratio. Participants will be randomly allocated in a 1:1 ratio to either intervention or control group arms, without stratification or minimisation. Randomisation numbers will be computer generated and assigned in strict sequence, using a secure, web-based randomisation system (CS RANDOM module, Clinsight Software). Randomisation will be managed by the Clinical Research Unit of Montpellier University Hospital, France, independently from the investigators. All screened subjects will be identifiable throughout the study by a unique subject number.

Eligible patients will be randomised into 2 groups (Fig. 1):

- Group 1: intervention group, e.g. patients participating in the cardiac rehabilitation program.

- Group 2: control group. These patients will have a regular non-modified follow-up with no rehabilitation program during the 12 -month study period. However, they will be able to participate in the cardiac rehabilitation program if they wish, once the 12 -month study period is over.

\subsection{Setting}

Overall, 16 CHD centres and 7 cardiac rehabilitation centres in France will participate in the study (Supplementary Fig. 1). Throughout the country, a total of 33 investigators will be in charge of patient recruitment. These investigators are paediatric cardiologists ( $n=16)$, adult congenital cardiologists $(n=3)$ or both $(n=14)$. In order to best represent the general CHD population, patients will be recruited in doctor's offices, private clinics, tertiary care public institutions, and university centres labelled by health authorities as referral centres for complex congenital heart diseases (M3C national health network). Participating cardiac rehabilitation centres are either paediatric $(n=1)$ or adult $(n=6)$ centres. All rehabilitation centres have expertise in exercise training and education in young patients with CHD [28].

Conduct of the study will be led by a local principal investigator (supported, when necessary, by a co-investigator), a research nurse or fellow, and a clinical research assistant, all of whom are trained in Good Clinical Practice and in the requirements of the study protocol. Each site will be responsible for the recruitment and scheduled followup visits of participants. 


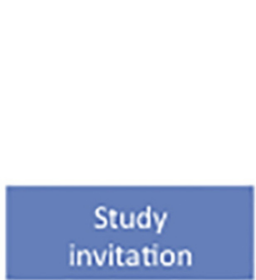

Recruitment

Potential partícipants attend baseline visit to give written

informed consent, confirm

eligibility and perform baseline assessments

No response, negative response, ineligible patient
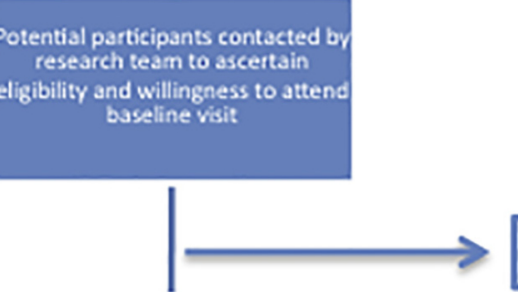

No response, negative response, ineligible patient

\section{(2)}
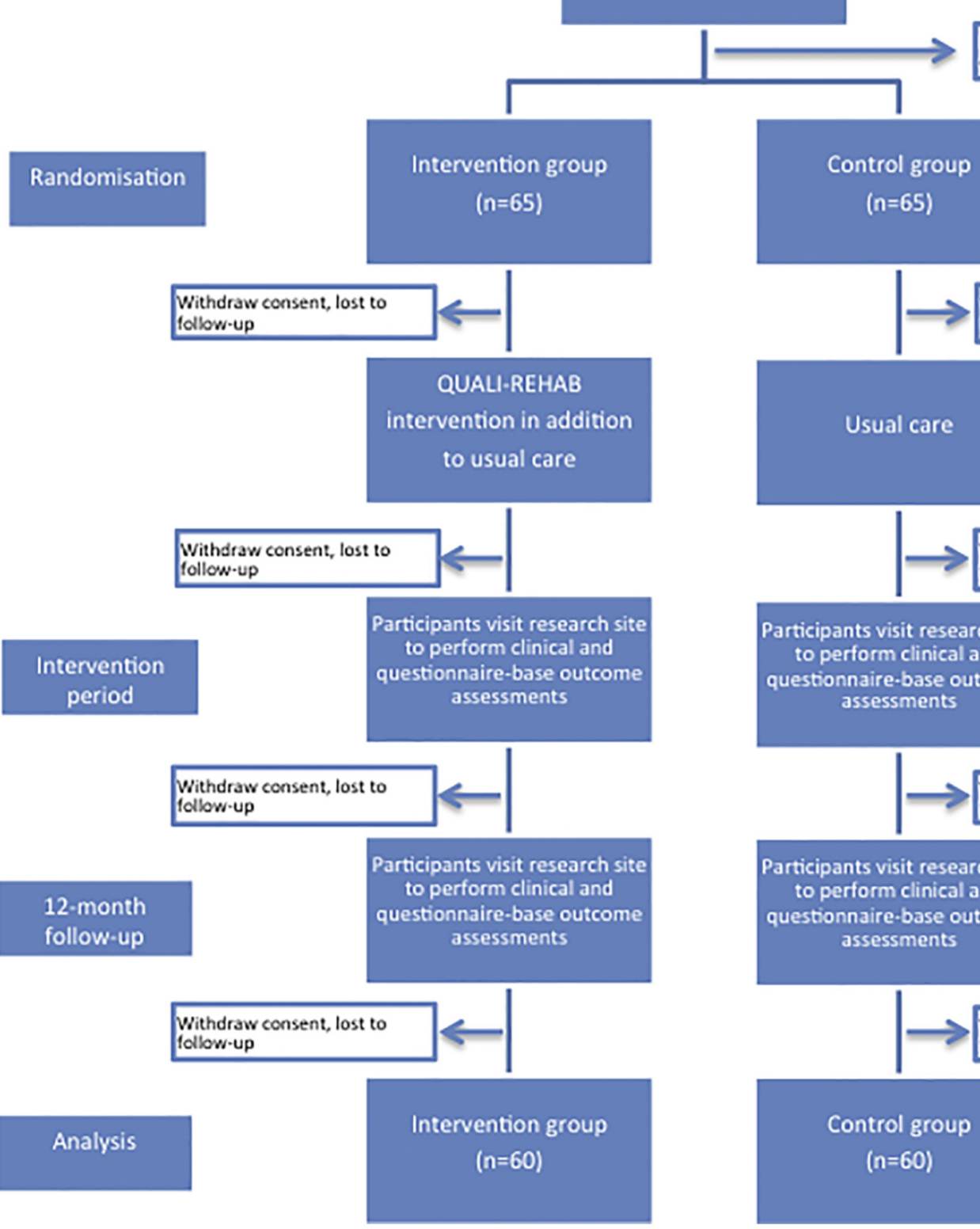

Ineligible patient, decline

consent
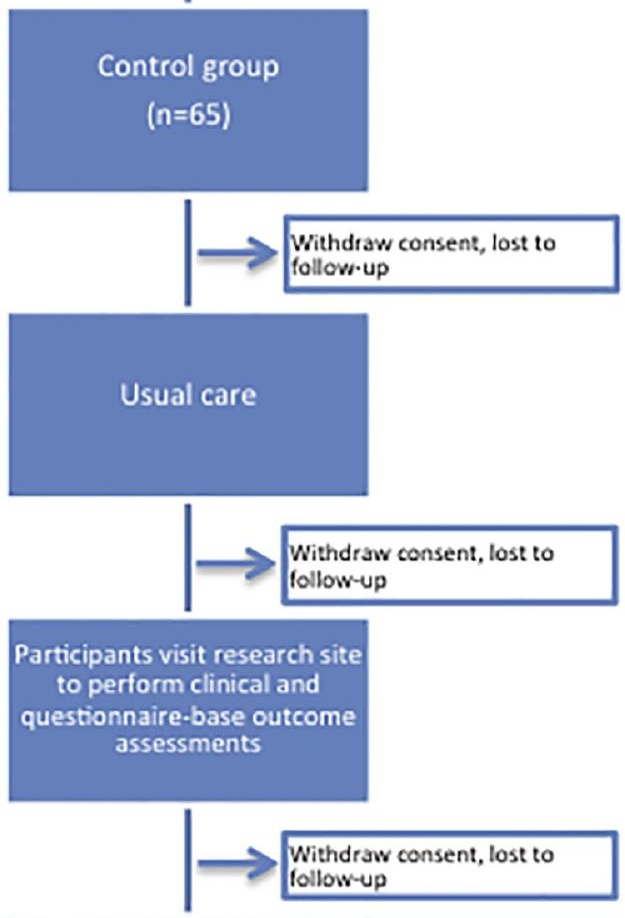

Participants visit research site

to perform clinical and

questionnaire-base outcome

assessments

Fig. 1. Flow chart. 


\subsection{Funding}

Montpellier University Hospital is the sponsor of the QUALI-REHAB trial. The study was funded after winning the 2017 call for proposal from the French Department of Health (DGOS-GIRCI-SOHO-PHRC-IR-2017). Additional research funding was obtained with student grants from the French Society of Cardiology (AG), the French Federation of Cardiology $(\mathrm{CB})$, and the APARD healthcare provider company $(\mathrm{CB})$. Donations (training bikes, wrist-based heart rate monitors, etc.) were obtained from the patient organisations "Association Petit Coeur De Beurre", "Association Pieces Jaunes" and "Association Astrid MRCPI".

\subsection{Study population}

Patients with a CHD, as defined by the international ACC-CHD classification [29], and aged from 13 to 25 years old, will be prospectively recruited in the participating centres, during an outpatient visit. Patients with a recent ( $<3$ months) medical check-up including a cardiology consultation, an ECG, an echocardiography and a cardio-pulmonary exercise test (CPET), as detailed in the current European guidelines [30], will be screened. Only patients with an abnormal CPET, with a $\mathrm{VO} 2_{\max }<80 \%$ of predicted $\mathrm{VO} 2_{\max }$ and/or a VAT $<55 \%$ of predicted $V O 2_{\max }$, will be eligible. Detailed inclusion and exclusion criteria are reported in Table 1.

\subsection{Intervention}

The QUALI-REHAB cardiac rehabilitation program includes a combined centre and home-based training design, with an overall duration of 12 weeks. The same program will be used in all rehabilitation centres, with harmonisation meetings prior to the start of the study. The home-based program will be carried-out by a single nation-wide health-provider company, partner of the research, and selected after a national call for tenders from the sponsor.

The rehabilitation program is detailed as follows (Supplementary Fig. 2):

- Day 1 to day 5: hospitalisation in the rehabilitation centre:

O Admission (day 1): definition of objectives with medical and nursing staff, personalized education report, clinical examination, and CPET.

O Exercise training program (day 1 to day 5 ):

- Daily group-sessions of interval training exercise at VAT level, using a stationary bicycle and outdoor group aerobic activities, supervised by a physical education teacher, in daily group sessions, of $1 \mathrm{~h}$ each (Fig. 2) [8].

Table 1

Trial entry.

Inclusion criteria

- Male or female aged 13 to 25 years old

- Patients with a CHD, as defined by the international ACC-CHD classification

- CPET performed within the last 3 months with $\mathrm{VO}_{\max }<80 \%$ of predicted $\mathrm{VO}_{\max }$ or $\mathrm{VAT}<55 \%$ of predicted $\mathrm{VO} 2_{\max }$

- Written informed consent for adult patients, or legal guardians for teenagers, and formal assent for teenagers

Exclusion criteria

- Absolute contraindications for CPET: fever, uncontrolled asthma, respiratory failure, acute myocarditis or pericarditis, uncontrolled arrhythmias causing symptoms or haemodynamic compromise, uncontrolled heart failure, acute pulmonary embolus or pulmonary infarction, and children with mental impairment leading to inability to cooperate

- Cardiac surgery planned during the study

- Patient who have undertaken cardiac rehabilitation within the last 24 months

- Uncontrolled arrhythmia

- Advanced atrioventricular block

- Uncontrolled heart failure (NYHA IV)

- Uncontrolled hypertension

- Acute myocarditis and pericarditis

- Symptomatic aortic stenosis

- Severe hypertrophic obstructive cardiomyopathy

- Acute systemic illness

- Recent (<3 months) intracardiac thrombus, embolism, or thrombophlebitis

- Pregnancy

- Severe musculoskeletal abnormalities

- Patients who are unable to understand the study information or unable to complete study procedures

- Patients with a severe intellectual disability that does not allow the completion of the quality of life questionnaire

- Patients who are in a long-term care institution or who are unwilling or unable to travel to research assessments or accommodate home visits

- Patients considered being unable to participate in the study for any other reason (for example, psychiatric disorder, dementia, life-threatening comorbidity, etc.)

- Patients participating in concurrent interventional research which may overburden the patient or confound data collection
- Education program (both individual and group sessions): multidisciplinary management (psychologist, dietician, and specialist nurse), following general cardiovascular disease prevention guidelines and specific issues in transition education programs for adolescents and young adults with CHD [28].

- Physical therapy: 1 individual session per day with a physiotherapist, including stretching, resistance, breathing, and thoracic extension exercises.

- Home-based training program (week 2 to week 12): 2 individual exercise sessions per week, of $1 \mathrm{~h}$ each, using a stationary bicycle, with the same interval-training exercise at VAT level (Fig. 2), supervised by a physical education teacher, with motivation reinforcement of patient's leisure physical activity. The research grant will cover the purchase of the stationary bikes, which will be delivered at home by the health provider.

- Three recall sessions at the rehabilitation centre, for 1 day each, every three weeks (between week 4 and 5 , between week 8 and 9 , and at week 12), including:

O Interval-training exercise at VAT level, using a stationary bicycle, supervised by a physical education teacher, in a 1-h group session (Fig. 2) [8].

- Reinforced education support: individual or group session with the specialist nurse.

O Physical therapy: 1 individual session with a physiotherapist, including stretching, resistance, breathing, and thoracic extension exercises.

- End of program at the rehabilitation centre, for 1 day (end of week 12): final evaluation with similar outcomes as of day 1 (including a CPET).

\subsection{Sample size}

We aim to recruit 130 patients ( 65 intervention: 65 control). The primary outcome is the change in the self-reported quality of life score with the PedsQL instrument. We used data from our previous quality of life cross-sectional studies in patients with $\mathrm{CHD}$ to calculate the sample size $[3,5,31,32]$. In our experience, as well as in similar studies using patient related outcomes, a difference of $<5$ points seems irrelevant and a difference of $>10$ points is ideal, but rarely obtained in clinical trials [31,33]. Therefore, we hypothesized to observe an increase in the overall quality of life score of $7 \pm 13.5$ points (over 100). With a $80 \%$ power, a bilateral alpha risk of $5 \%$, and potentially $10 \%$ of loss to follow-up and/or missing data on the primary outcome, we need to include 65 patients in the group 1 and 65 patients in the group 2 .

\subsection{Statistical analysis}

All included subjects will be considered in the description of the population (baseline characteristics). An intention-to-treat analysis will be used, and each randomised subject will be analysed in his/her treatment arm. A per-protocol analysis, including all randomised subjects with a valid primary efficacy measurement and with no important protocol deviation (patients who have successfully completed the rehabilitation, with at least $80 \%$ of the sessions) that could affect the evaluation of the main outcome, will also be carried out for parameters to study mechanisms of action.

A description of each group will be made by giving the frequencies of the different categories for the qualitative variables. In case of non-comparability of the groups on one of the confounding factors, an adjustment or stratification (in case of interaction) will be considered.

\subsection{Main outcome}

The main outcome is the variation of the PedsQL self-reported quality of life score, between baseline (M0) and 12-month follow-up (M12). The PedsQL generic quality of life questionnaire has four multidimensional scales: physical functioning ( 8 items), emotional functioning ( 5 items), social functioning ( 5 items), school functioning ( 5 items). The three summary scores are: total scale score ( 23 items), physical health summary score ( 8 items), psychosocial health summary score ( 15 items). Each item uses a 5-point Likert scale from 0 (never) to 4 (almost always). Items are reversed scored and linearly transformed to a 0 100 scale, higher scores indicating a better quality of life. Psychometric properties showed reliability, validity and responsiveness to clinical change over time [34]. After translation and cultural adaptation, the psychometric properties of the French version of the PedsQL appeared to be acceptable [35]. Two versions of the PedsQL questionnaire (13-18 and $18-25$ years old) will be used for adolescents and young adults, respectively.

\subsection{Secondary outcomes}

The following outcomes will be measured at baseline (M0) and 12-month follow-up (M12):

- CPET variables: maximum oxygen uptake $\left(\mathrm{VO}_{\max }\right)$; ventilatory anaerobic threshold (VAT); ventilator efficiency (VE/VCO2 slope), oxygen uptake efficiency slope (OUES), and oxygen pulse (VO2/heart rate). As detailed in our previous multicentre CPET studies, exercise test procedures in all participating laboratories will be harmonized before the start of the study [4,5]. All centres will use the same CPET cycle ergometer protocol, to obtain a homogeneous incremental overall duration between 8 and 12 min: a 1-minute rest; a 3-minute warm-up (10 to 20 watts) in increments of 10,15 , or 20 watts each minute; a pedalling rate of 60 to $80 \mathrm{rpm}$; a 3-minute active recovery ( 20 watts); and a 2-minute rest. The CPET will be considered as maximal when 3 out of the 4 following criteria will be reached: respiratory exchange ratio $(\mathrm{RER}=\mathrm{VCO} 2 / \mathrm{VO} 2) \geq 1$, maximum heart rate $>85 \%$ of maximal age-predicted heart rate, limit of the patient's tolerance despite verbal encouragement, plateau of $\mathrm{VO} 2\left(\mathrm{VO}_{\max }\right)$ despite the increasing exercise intensity, and patient's 


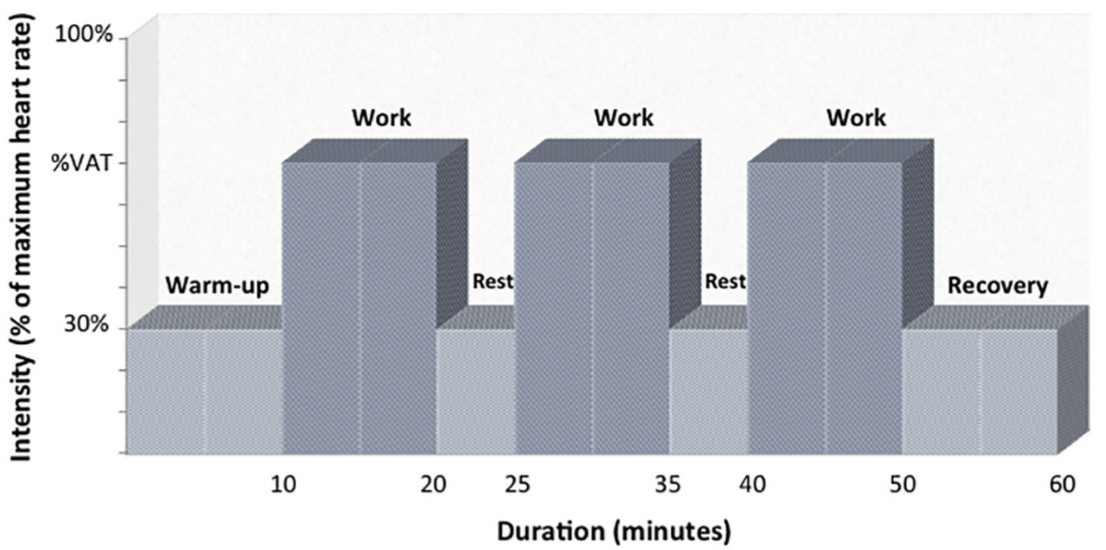

Fig. 2. Interval training session. Legend: \%VAT, heart rate at ventilatory anaerobic threshold, expressed as a percentage of the maximum heart rate.

inability to provide a minimum pedalling frequency of 60 per minute despite verbal encouragement. The VAT will be estimated using Beaver's method [36]. VO2 ${ }_{\max }$ and VAT values will be normalized in a percentage of the predicted $\mathrm{VO}_{\mathrm{max}}$ using reference values for cycle ergometer test in the general paediatric and adult population [37,38].

- The peak exercise stroke volume measured during CPET with a non-invasive impedance cardiograph device (PhysioFlow $($ ) $[39]$.

- The level of physical activity with the Ricci and Gagnon questionnaire, composed by 8 items (total score < 16 points: no activity; 17 to 32 points: moderate activity; 33 to 40 points: intensive activity) [40].

- The level of knowledge with the Leuven knowledge questionnaire for CHD [41].

- The clinical outcomes: NYHA functional class, blood pressure, body mass index (BMI), healthcare usage (primary and secondary care contacts, hospitalisation), and medication.

- The level of anxiety with the self-administered State and Trait Anxiety Inventory (STAI) questionnaire for young adults and the STAI-Children questionnaire for adolescents [42].

- The level of depression with the self-administered Beck Depression Inventory (BDI) questionnaire for young adults and the Child Depression Inventory (CDI) questionnaire for adolescents $[43,44]$

- The parents-reported quality of life score with the proxy version of the PedsQL for adolescents (aged 13-18 years old) [34].

- The socio-economic status of the patients and/or their family (only at baseline)

- The safety outcomes.

- The acceptability of the intervention to participants.

All outcomes are reported in Table 2.

2.10. Ethics

The study will be conducted in compliance with the Good Clinical Practices protocol and Declaration of Helsinki principles. It was approved by a drawn National Ethics

Table 2

Outcome measures.

Primary outcome

- Quality of life score: PedsQL self-questionnaire (version 13-18 years for adolescents and version $18-25$ years for young adults)

Secondary outcomes

- CPET variables

O $\mathrm{VO}_{\max }$

O VAT

O VE/VCO2 slope

O OUES (oxygen uptake efficiency slope)

O Oxygen pulse

- Peak exercise stroke volume (Physioflow ${ }^{\circledR}$ )

- Level of physical activity (Ricci and Gagnon questionnaire)

- Level of knowledge (Leuven knowledge CHD questionnaire)

- Clinical outcomes: NYHA functional class, blood pressure, body mass index (BMI), healthcare usage (primary and secondary care contacts, hospitalisation), and medication

- Level of anxiety (STAI self-questionnaire for young adults and the STAI-Children self-questionnaire for adolescents)

- Level of depression (BDI self-questionnaire for young adults and CDI selfquestionnaire for adolescents)

- Proxy version of the PedsQL for parents of adolescents (aged 13-18 years old)

- The socio-economic status of the patient and/or the family (only at baseline)

- Safety outcomes

- Acceptability of the intervention to participants
Committee (North-West I-2018-A00874-51) and registered on Clinicaltrials.gov (NCT03690518). As required by the French Department of Health, all participating cardiac rehabilitation centres have received agreement from the regional health authorities. Informed consent will be obtained from all patients and their parents or legal guardians for minors.

\section{Expected results and perspectives}

In the continuity of our research on quality of life in patients with CHD $[3,5,31,32,45]$, we expect to observe an improvement of healthrelated quality of life in patients undergoing the rehabilitation program. From our perspective, considering the quality of life as the main outcome in the QUALI-REHAB trial is both original and primordial in the young CHD population. Indeed, measuring patient-reported outcomes (PRO) in CHD clinical trials is relevant [2], considering that these young patients experience a low level of physical impairment during childhood, but an increased cardio-vascular risk during adulthood [33]. In line with the European Medicines Agency (EMA) promotion of PRO measures in clinical research, most drug trials currently evaluate the quality of life as a secondary outcome [46]. However, further research from randomised trials remains necessary, when considering quality of life assessment as a primary outcome.

The cardiopulmonary fitness, as assessed by CPET, is moderately impaired in young patients with CHD $[4,6]$, and the effect of rehabilitation programs on $\mathrm{VO}_{\max }$ and VAT in this population remains controversial, as a result of a lack of randomised studies [21,22,27]. However, exercise capacity in the contemporary adult CHD population is significantly impaired [47]. Therefore, we hope that this rehabilitation program will contribute to reverse the vicious circle of deconditioning in young patients with CHD, and, ultimately participate in reducing the cardiovascular risks related to inactivity in this population. From a general perspective, proposing a rehabilitation program for this generation of CHD patients may respond to both the epidemiology and current public health challenges in the field of congenital cardiology $[1,28]$. Indeed, when physical deconditioning is diagnosed and managed at an early stage in chronic diseases, participation in rehabilitation and education interventions stand a as chance of reducing morbidity and mortality [48].

Classically, centre-based cardiac rehabilitation programs dedicated to adult patients with heart failure include 3 weeks at the hospital, and, therefore, are less adapted to youth with CHD. The QUALI-REHAB trial draws on "modern" supervised home-based rehabilitation programs [17], but also includes centre-based supervision, with an initial 5-day period at the hospital to initiate exercise training and patient education, and 2 days of recall in the institution, in order to maintain patient motivation.

Furthermore, the QUALI-REHAB program takes into account the importance of therapeutic education, as a factor favouring behaviour 
modification, and contributing to the overall success of rehabilitation [49]. The main educational objective is to bring more autonomy to this youth population in transition to adulthood, not only in the knowledge of their CHD, but also in every domain of their life (e.g. physical activity, psychological well-being, sexuality, graduate studies, professional carriers, risky behaviour, etc.).

The QUALI-REHAB study participates in the promotion of developing expertise in CHD in cardiac rehabilitation centres, while responding to the contemporary society's request for outpatient care. If the QUALIREHAB trial is positive, combined centre and home-based rehabilitation programs could be generalized to a larger CHD population, as well as youth with other chronic diseases, in the perspective of health prevention, follow-up optimization, and socio-economic benefit [48].

Ultimately, such programs may contribute to reduce overall morbidity and mortality, and improve quality of life in the long term [50].

\section{Conclusion}

With a nationwide multicentre randomised study, the QUALI-REHAB trial aims to assess the impact of a combined centre and home-based cardiac rehabilitation program on the quality of life of adolescents and young adults with CHD. The originality of the QUALI-REHAB trial lies in a design particularly suited to a youth generation, using a combined supervised training program at home and at the hospital, but also giving an important place to patient education.

\section{Acknowledgement of grant support}

The study was funded by the French Department of Health (DGOSGIRCI-SOHO-PHRC-IR-2017), by a research grant from the French Society of Cardiology, by a research grant from the French Federation of Cardiology, and by a research grant from APARD healthcare provider company.

\section{Declarations of interest}

None.

\section{Acknowledgments}

The authors are grateful to Ms. Anne Cadene, Odile Bardet, Chloe Bureau and Amandine Marquina for their administrative and organizational support. We also thank the patient organisations "Association Petit Coeur De Beurre", "Association Pieces Jaunes" and "Association Astrid MRCPI" for their donations.

\section{References}

[1] P. Khairy, R. Ionescu-Ittu, A.S. Mackie, M. Abrahamowicz, L. Pilote, A.J. Marelli, Changing mortality in congenital heart disease, J. Am. Coll. Cardiol. 56 (2010) $1149-1157$.

[2] P. Moons, A.H. Kovacs, K. Luyckx, C. Thomet, W. Budts, J. Enomoto, et al., Patientreported outcomes in adults with congenital heart disease: inter-country variation, standard of living and healthcare system factors, Int. J. Cardiol. 251 (2018) 34-41.

[3] P. Amedro, R. Dorka, S. Moniotte, S. Guillaumont, A. Fraisse, B. Kreitmann, et al., Quality of life of children with congenital heart diseases: a multicenter controlled cross-sectional study, Pediatr. Cardiol. 36 (2015) 1588-1601.

[4] P. Amedro, A. Gavotto, S. Guillaumont, H. Bertet, M. Vincenti, G. De La Villeon, et al., Cardiopulmonary fitness in children with congenital heart diseases versus healthy children, Heart 104 (2018) 1026-1036.

[5] P. Amedro, M.C. Picot, S. Moniotte, R. Dorka, H. Bertet, S. Guillaumont, et al., Correlation between cardio-pulmonary exercise test variables and health-related quality of life among children with congenital heart diseases, Int. J. Cardiol. 203 (2016) 1052-1060.

[6] J. Muller, B. Bohm, S. Semsch, R. Oberhoffer, J. Hess, A. Hager, Currently, children with congenital heart disease are not limited in their submaximal exercise performance, Eur. J. Cardiothorac. Surg. 43 (2013) 1096-1100.
[7] F. Moola, C. Fusco, J.A. Kirsh, The perceptions of caregivers toward physical activity and health in youth with congenital heart disease, Qual. Health Res. 21 (2011) 278-291.

[8] T. Takken, A. Giardini, T. Reybrouck, M. Gewillig, H.H. Hovels-Gurich, P.E. Longmuir, et al., Recommendations for physical activity, recreation sport, and exercise training in paediatric patients with congenital heart disease: a report from the Exercise, Basic \& Translational Research Section of the European Association of Cardiovascular Prevention and Rehabilitation, the European Congenital Heart and Lung Exercise Group, and the Association for European Paediatric Cardiology, Eur. J. Prev. Cardiol. 19 (2012) 1034-1065.

[9] P.E. Longmuir, J.A. Brothers, S.D. de Ferranti, L.L. Hayman, G.F. Van Hare, G.P. Matherne, et al., Promotion of physical activity for children and adults with congenital heart disease: a scientific statement from the American Heart Association, Circulation 127 (2013) 2147-2159.

[10] T. Papasavvas, M. Alhashemi, D. Micklewright, Association between depressive symptoms and exercise capacity in patients with heart disease: a meta-analysis, J Cardiopulm Rehabil Prev. 37 (2017) 239-249.

[11] C. Karsenty, P. Maury, N. Blot-Souletie, M. Ladouceur, B. Leobon, V. Senac, et al., The medical history of adults with complex congenital heart disease affects their social development and professional activity, Arch. Cardiovasc. Dis. 108 (2015) 589-597.

[12] C. Tamayo, C. Manlhiot, K. Patterson, S. Lalani, B.W. McCrindle, Longitudinal evaluation of the prevalence of overweight/obesity in children with congenital heart disease, Can. J. Cardiol. 31 (2015) 117-123.

[13] F. Moola, B.W. McCrindle, P.E. Longmuir, Physical activity participation in youth with surgically corrected congenital heart disease: devising guidelines so Johnny can participate, Paediatr. Child Health 14 (2009) 167-170.

[14] R.S. Taylor, V.A. Sagar, E.J. Davies, S. Briscoe, A.J. Coats, H. Dalal, et al., Exercise-based rehabilitation for heart failure, Cochrane Database Syst. Rev. (4) (2014) 1-121 CD003331.

[15] G. Kwan, G.J. Balady, Cardiac rehabilitation 2012: advancing the field through emerging science, Circulation 125 (2012) e369-e373.

[16] M.F. Piepoli, S. Binno, U. Corra, P. Seferovic, V. Conraads, T. Jaarsma, et al., ExtraHF survey: the first European survey on implementation of exercise training in heart failure patients, Eur. J. Heart Fail. 17 (2015) 631-638.

[17] V. Eyre, C.C. Lang, K. Smith, K. Jolly, R. Davis, C. Hayward, et al., Rehabilitation enablement in chronic heart failure-a facilitated self-care rehabilitation intervention in patients with heart failure with preserved ejection fraction (REACH-HFpEF) and their caregivers: rationale and protocol for a single-centre pilot randomised controlled trial, BMJ Open 6 (2016), e012853. .

[18] K. Gierat-Haponiuk, I. Haponiuk, D. Szalewska, M. Chojnicki, R. Jaworski, P. Niedoszytko, et al., Effect of complex cardiac rehabilitation on physical activity and quality of life during long-term follow-up after surgical correction of congenital heart disease, Kardiol. Pol. 73 (2015) 267-273.

[19] J.S. Dua, A.R. Cooper, K.R. Fox, Stuart A. Graham, Exercise training in adults with congenital heart disease: feasibility and benefits, Int. J. Cardiol. 138 (2010) 196-205.

[20] K. Dulfer, N. Duppen, I.M. Kuipers, M. Schokking, R.T. van Domburg, F.C. Verhulst, et al., Aerobic exercise influences quality of life of children and youngsters with congenital heart disease: a randomized controlled trial, J. Adolesc. Health 55 (2014) 65-72.

[21] M. Gomes-Neto, M.B. Saquetto, C.M. da Silva Silva e, C.S. Conceicao, V.O. Carvalho, Impact of exercise training in aerobic capacity and pulmonary function in children and adolescents after congenital heart disease surgery: a systematic review with meta-analysis, Pediatr. Cardiol. 37 (2016) 217-224.

[22] N. Duppen, T. Takken, M.T. Hopman, A.D. ten Harkel, K. Dulfer, E.M. Utens, et al., Systematic review of the effects of physical exercise training programmes in children and young adults with congenital heart disease, Int. J. Cardiol. 168 (2013) 1779-1787.

[23] N. Duppen, L.M. Geerdink, I.M. Kuipers, S.S. Bossers, L.P. Koopman, A.P. van Dijk, et al., Regional ventricular performance and exercise training in children and young adults after repair of tetralogy of Fallot: randomized controlled pilot study, Circ. Cardiovasc. Imaging 8 (2015).

[24] T. van der Bom, M.M. Winter, J.L. Knaake, E. Cervi, L.S. de Vries, A. Balducci, et al., Long-term benefits of exercise training in patients with a systemic right ventricle, Int. J. Cardiol. 179 (2015) 105-111.

[25] R.L. Cordina, S. O'Meagher, A. Karmali, C.L. Rae, C. Liess, G.J. Kemp, et al., Resistance training improves cardiac output, exercise capacity and tolerance to positive airway pressure in Fontan physiology, Int. J. Cardiol. 168 (2013) 780-788.

[26] M.M. Winter, B.J. Bouma, A.P. van Dijk, M. Groenink, P.T. Nieuwkerk, M.N. van der Plas, et al., Relation of physical activity, cardiac function, exercise capacity, and quality of life in patients with a systemic right ventricle, Am. J. Cardiol. 102 (2008) $1258-1262$.

[27] A.U. Tikkanen, A.R. Oyaga, O.A. Riano, E.M. Alvaro, J. Rhodes, Paediatric cardiac rehabilitation in congenital heart disease: a systematic review, Cardiol. Young 22 (2012) 241-250.

[28] C. Sable, E. Foster, K. Uzark, K. Bjornsen, M.M. Canobbio, H.M. Connolly, et al., Best practices in managing transition to adulthood for adolescents with congenital heart disease: the transition process and medical and psychosocial issues: a scientific statement from the American Heart Association, Circulation 123 (2011) $1454-1485$.

[29] L. Houyel, B. Khoshnood, R.H. Anderson, N. Lelong, A.C. Thieulin, F. Goffinet, et al., Population-based evaluation of a suggested anatomic and clinical classification of congenital heart defects based on the International Paediatric and Congenital Cardiac Code, Orphanet J. Rare Dis. 6 (2011) 64.

[30] H. Baumgartner, P. Bonhoeffer, N.M. De Groot, F. de Haan, J.E. Deanfield, N. Galie, et al., ESC guidelines for the management of grown-up congenital heart disease (new version 2010), Eur. Heart J. 31 (2010) 2915-2957. 
[31] P. Amedro, F. Bajolle, H. Bertet, R. Cheurfi, D. Lasne, E. Nogue, et al., Quality of life in children participating in a non-selective INR self-monitoring VKA-education programme, Arch. Cardiovasc. Dis. 111 (2018) 180-188.

[32] P. Amedro, N. Tahhan, H. Bertet, C. Jeandel, S. Guillaumont, T. Mura, et al., Healthrelated quality of life among children with turner syndrome: controlled crosssectional study, J. Pediatr. Endocrinol. Metab. 30 (2017) 863-868.

[33] S.D. Anker, S. Agewall, M. Borggrefe, M. Calvert, J. Jaime Caro, M.R. Cowie, et al., The importance of patient-reported outcomes: a call for their comprehensive integration in cardiovascular clinical trials, Eur. Heart J. 35 (2014) 2001-2009.

[34] J.W. Varni, M. Seid, P.S. Kurtin, PedsQL 4.0: reliability and validity of the Pediatric Quality of Life Inventory version 4.0 generic core scales in healthy and patient populations, Med. Care 39 (2001) 800-812.

[35] S. Tessier, A. Vuillemin, J.-L. Lemelle, S. Briancon, Psychometric properties of the French Pediatric Quality of Life Inventory Version 4.0 (PedsQL ${ }^{\mathrm{TM}} 4.0$ ) generic core scales, Eur. Rev. Appl. Psychol. 59 (2009) 291-300.

[36] W.L. Beaver, K. Wasserman, B.J. Whipp, A new method for detecting anaerobic threshold by gas exchange, J. Appl. Physiol. 60 (1985) 2020-2027.

[37] D.M. Cooper, C. Berry, N. Lamarra, K. Wasserman, Kinetics of oxygen uptake and heart rate at onset of exercise in children, J Appl Physiol 59 (1985) 211-217.

[38] D.M. Cooper, D. Weiler-Ravell, B.J. Whipp, K. Wasserman, Aerobic parameters of exercise as a function of body size during growth in children, J. Appl. Physiol. Respir. Environ. Exerc. Physiol. 56 (1984) 628-634.

[39] A. Legendre, D. Bonnet, L. Bosquet, Reliability of peak exercise stroke volume assessment by impedance cardiography in patients with residual right outflow tract lesions after congenital heart disease repair, Pediatr. Cardiol. 39 (2018) 45-50.

[40] A. Vuillemin, G. Denis, F. Guillemin, C. Jeandel, A review of evaluation questionnaires for physical activity, Rev. Epidemiol. Sante Publique 46 (1998) 49-55.

[41] H.L. Yang, Y.C. Chen, J.K. Wang, B.S. Gau, C.W. Chen, P. Moons, Measuring knowledge of patients with congenital heart disease and their parents: validity of the 'Leuven
Knowledge Questionnaire for Congenital Heart Disease', Eur. J. Cardiovasc. Nurs. 11 (2012) 77-84.

[42] P.C. Kendall, A.J. Finch Jr., S.M. Auerbach, J.F. Hooke, P.J. Mikulka, The state-trait anxiety inventory: a systematic evaluation, J. Consult. Clin. Psychol. 44 (1976) 406-412.

[43] V.C. Delisle, A.T. Beck, R.C. Ziegelstein, B.D. Thombs, Symptoms of heart disease or its treatment may increase Beck depression inventory scores in hospitalized postmyocardial infarction patients, J. Psychosom. Res. 73 (2012) 157-162.

[44] W.J. Helsel, J.L. Matson, The assessment of depression in children: the internal structure of the Child Depression Inventory (CDI), Behav. Res. Ther. 22 (1984) 289-298.

[45] P. Amedro, A. Basquin, V. Gressin, P. Clerson, X. Jais, J.B. Thambo, et al., Healthrelated quality of life of patients with pulmonary arterial hypertension associated with CHD: the multicentre cross-sectional ACHILLE study, Cardiol. Young 26 (2016) 1250-1259.

[46] G. Apolone, G. De Carli, M. Brunetti, S. Garattini, Health-related quality of life (HR$\mathrm{QOL}$ ) and regulatory issues. An assessment of the European Agency for the Evaluation of Medicinal Products (EMEA) recommendations on the use of HR-QOL measures in drug approval, PharmacoEconomics 19 (2001) 187-195.

[47] A. Kempny, K. Dimopoulos, A. Uebing, P. Moceri, L. Swan, M.A. Gatzoulis, et al., Reference values for exercise limitations among adults with congenital heart disease. Relation to activities of daily life-single centre experience and review of published data, Eur. Heart J. 33 (2012) 1386-1396.

[48] F.W. Booth, C.K. Roberts, M.J. Laye, Lack of exercise is a major cause of chronic diseases, Compr. Physiol. 2 (2012) 1143-1211.

[49] P.E. Longmuir, P.N. Tyrrell, M. Corey, G. Faulkner, J.L. Russell, B.W. McCrindle, Homebased rehabilitation enhances daily physical activity and motor skill in children who have undergone the Fontan procedure, Pediatr. Cardiol. 34 (2013) 1130-1151.

[50] J.R. Santos-Parker, T.J. LaRocca, D.R. Seals, Aerobic exercise and other healthy lifestyle factors that influence vascular aging, Adv. Physiol. Educ. 38 (2014) 296-307. 